\title{
Covering a Robot Fingertip with uSkin: a Soft Electronic Skin with Distributed 3-axis Force Sensitive Elements for Robot Hands
}

\author{
Tito Pradhono Tomo ${ }^{1}$, Alexander Schmitz ${ }^{1}$, Wai Keat Wong ${ }^{1}$, Harris Kristanto ${ }^{1}$, \\ Sophon Somlor ${ }^{1}$, Jinsun Hwang ${ }^{1}$, Lorenzo Jamone ${ }^{2}$, and Shigeki Sugano ${ }^{1}$
}

\begin{abstract}
Distributed tactile sensing is crucial to perform stable, subtle, and precise manipulation so that a robot can recognize and handle objects properly. However, currently existing skin sensors still have common problems such as complex and expensive production or are difficult to integrate into robot hands. In particular, a practical distributed soft skin sensor system that can cover various parts of the robot hand, measure force in 3axis, with a subcentimeter spatial density, and digital output at the same time does not exist yet. This paper discusses uSkin, a soft, distributed, 3-axis force sensor for robot hands and presents its implementation for multi-curved fingertips. The sensor is lowcost, easy to manufacture, and can measure normal and shear forces. The experimental results revealed that this sensor has $10 \%$ hysteresis for perpendicular force with a maximum range of $6 \mathrm{~N}$. The Signal to Noise Ratio (SNR) value of $54 \mathrm{~dB}$ for 0.4 $\mathrm{N}$ load was achieved, which constitutes the state of the art for this kind of sensors. Evaluation experiments also showed that the distributed 3-axis load cells could produce vectors that represent the shape of objects. This opens the possibility that the sensor can be used for classifying different shapes. Furthermore, the fingertip sensor was installed on the Allegro hand and the changing force measurements when the robot is grasping an object are presented.
\end{abstract}

Index Terms-Force and Tactile Sensing, Dexterous Manipulation, Multifingered Hands.

\section{INTRODUCTION}

$\mathbf{S}$ KIN for robot hands needs to fulfill various requirements to enable fine manipulation. Soft skin has been proven effective to increase the object manipulation performance in robots. As stated in [1], by covering a fingertip with soft silicone, the in-hand manipulation performance becomes more stable. Moreover, many researchers have applied soft skin sensors on their humanoid robots for enhanced safety and object handling. For example, Twendy-One's hand can securely grasp various objects such as soft plastic or paper cups and freely manipulate small objects such as a pencil or straw [2]. Most distributed tactile sensors can measure only single axis force or have a low spatial density.

Manuscript received: February, 15, 2017; Revised June, 29, 2017; Accepted July, 14, 2017.

This paper was recommended for publication by Editor John Wen upon evaluation of the Associate Editor Antonio Bicchi and Reviewers' comments.

${ }^{1}$ Tito Pradhono Tomo, Wai Keat Wong, Alexander Schmitz, Harris Kristanto,Sophon Somlor and Shigeki Sugano are with Waseda University, Tokyo, Japan tito@toki.waseda.jp

${ }^{2}$ Lorenzo Jamone is with ARQ (Advanced Robotics at Queen Mary), School of Electronic Engineering and Computer Science, Queen Mary University of London, UK l . jamone@ qmul.ac.uk

Digital Object Identifier (DOI): see top of this page.
Sensors with a high spatial density are desirable for in-hand object manipulation or tactile servoing tasks [3]. However, a higher spatial density usually leads to a larger number of wire connections, bulky electronics, and crosstalk between the sensing cells. Considering these factors, the realization and integration of such a kind of sensor in a robot hand is a challenging task.

Furthermore, the capability to measure a distributed force vector is crucial for dexterous object handling and provides rich haptic information about the manipulated objects. Since the individual tri-axial load cells of our sensor are very small and close to each other, the sensor allows us to precisely retrieve i) the points of contact, ii) the 3D force vector at each point of contact, iii) the overall shape of the area of contact, iv) the overall $3 \mathrm{D}$ force vector applied to the area of contact. The sensor deals well with cases in which multiple contact points are simultaneously present on the same module: this is a typical problematic situation for current state of the art tri-axial force sensors.

Our lab has introduced a 3-axis capacitive based skin sensor in [4]. However, each 3-axis force measurement unit requires a volume of $14 \times 14 \times 7 \mathrm{~mm}$, resulting in a low spatial density; also making it difficult to cover a multi-curved shape, such as a fingertip, with this sensor. More recently, we introduced a single 3-axis Hall-effect based skin sensor including its characterization and SNR modeling in [5] and [6]. Subsequently, this sensor was upgraded into a flat distributed skin sensor for robot phalanges with $4 \times 4$ load cells (each 3 -axis) per module and the sensor was integrated into the Allegro Hand [7]. We named our sensor system "uSkin." While in the previous work flat surfaces could be covered, our final goal is to cover all parts of the hand. Fingertips have a multicurved shape which is difficult to cover with tactile skin sensors, but fingertips are especially important for fine manipulation. For example, fingertips are especially relevant during manipulating a small object [2]. A clear shortcoming of our previous work was that it was applied only to flat surfaces, but multicurved fingertips are beneficial [8]. In general, many soft skin sensors can be implemented for a flat or cylindrical surface, but only a few of them can be utilized to cover a multicurved surface [2] [9] [10]. The current paper will introduce uSkin for the Allegro hand's fingertip. The dimension of the fingertip including the silicone skin is within $30 \times 35 \times 28 \mathrm{~mm}$; each fingertip can measure 24 force vectors with 24 3-axis Hall-effect sensors (MLX90393). Additionally, each sensor also provides temperature measurements. An inte- 
gral part of the skin sensor is $4 \mathrm{~mm}$ thick silicone. The output of each module is digital and requires only nine wires while providing 24x4 (3-axis magnetic field and one temperature) data. Therefore, uSkin can be connected to a microcontroller directly through I2C (Inter-Integrated Circuit) protocol without needing to amplify the signal first. Our current design is based on the Allegro Hand's shape and size. However, in general, the shape of uSkin can be configured according to various robot hand forms.

To summarize, the main contributions of this work, both with respect to our previously published design and with respect to the literature, is that we can cover multicurved surfaces.

The rest of this paper is organized as follows. In Section II we provide a review of related tactile sensors for robotic fingertips. Section III describes the sensor principle, the production process, and the integration into the Allegro hand. Section IV presents the experimental procedure that was used to evaluate the sensor and shows the results. Section V draws conclusions and presents future work.

\section{RELATED WORKS}

The literature describes many tactile sensors [11] [12], but few of them can measure multi-axis force and can or have been integrated into robotic hands. Several robot hands have already implemented a distributed sensing in their fingertips, for example [2] [9] [10] [13]. However, those sensors can only provide one axis of force per load cell. Tri-axial sensors based on strain gauges were integrated into the soft skin of the robot Macra [14]. Because of the sensor size, using them for distributed sensing in robot hands may not be feasible.

Optical sensors have been used for distributed tactile sensing, as they can provide high spatial density. For example, GelSight has a high spatial density and it is possible to be integrated into a gripper [15]. However, integrating this sensor into an anthropomorphic hand that has multiple fingers to perform an in-hand manipulation task appears difficult. Another example is TacTip [16]. It can measure distributed 3axis force, texture, and is low in cost. However, the application appears to be limited to fingertip-only and cannot be used to cover other parts. The quantum tunneling composite (QTC) based fingertip sensor presented in [17] can also measure distributed 3-axis force. To measure a 3-axis force, this type of sensor requires a small dome while a flat surface skin is preferable.

Although many tactile skin sensors have been described in literature, only few are commercially available. Touchence ${ }^{1}$ sells a thin, small-sized 3-axis tactile sensor based on microelectromechanical systems (MEMS) piezoelectric elements, but the sensor is rigid, and the necessary additional electronics are bigger than the sensor itself. A smaller optical tri-axial sensor is currently available from OptoForce ${ }^{2}$. The sensor is $10 \mathrm{~mm}$ wide and $8 \mathrm{~mm}$ high. A robotic fingertip that is multi-modal and can measure distributed single-axis force

\footnotetext{
${ }^{1}$ http://www.touchence.jp/

${ }^{2}$ http://optoforce.com/
}

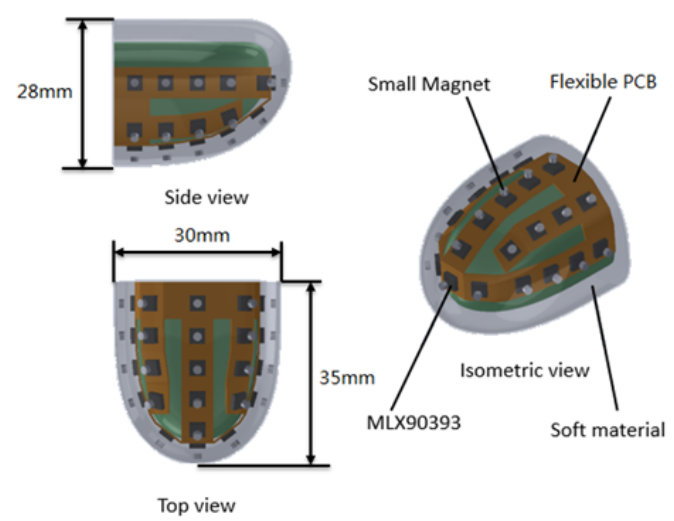

Fig. 1. Conceptual design of uSkin for a fingertip.

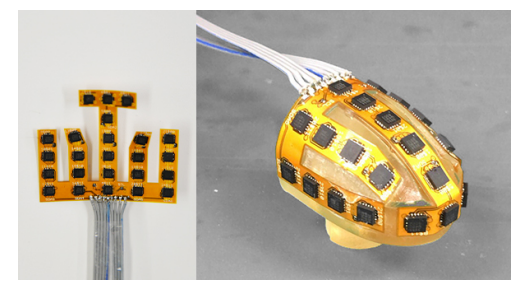

Fig. 2. A flexible PCB attached to the $3 \mathrm{D}$ printed fingertip.

is available from SynTouch ${ }^{3}$. However, this sensor is very expensive.

The idea of using Hall effect sensors and magnets to measure a tactile response was originally proposed in [18] and [19], where only preliminary prototypes were presented. The sensors in [20] [21] are integrated into a robot hand, but only normal forces can be measured. The work in [22] instead proposes a 3D sensor, but the sensors were not used for distributed sensing. The work described in [23] [24] has been successfully applied to real robotic scenarios; however, the design they proposed (with a rubber dome and four Hall effect sensors) imposed constraints on the minimum size of the whole system. The work in [25] integrates one 3-axis sensor per finger phalanx.

In summary, a practical distributed soft skin sensor system that can cover various parts of the robot hand, measure force in 3-axis, with a subcentimeter spatial density, and digital output at the same time does not exist yet. The system presented in this paper has all these features and is moreover low cost in terms of the required materials and labor effort.

\section{SENSOR DESCRIPTION}

In this section the structure and the manufacturing process of uSkin will be described. The whole production process is fast and effortless.

\section{A. Sensor Concept}

Our lab firstly developed uSkin for the Allegro Hand's phalanges in [7]. By converting magnetic field changes into forces, our sensor could measure normal and shear forces on

${ }^{3}$ https://www.syntouchinc.com/ 


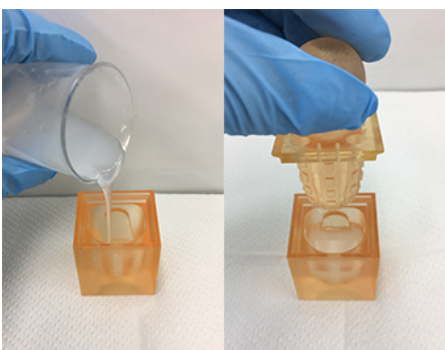

(a)

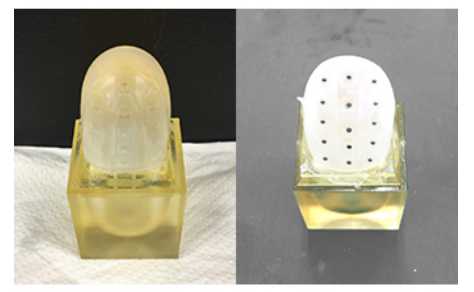

(b)

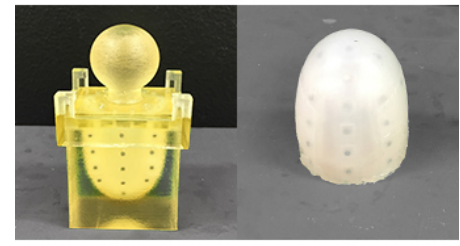

(c)

Fig. 3. (a) Liquid silicone rubber was poured, and the hole maker was placed (b) 24 small magnets were placed inside the holes. (c) Magnets were covered with another layer of silicone.

16 contact points per phalange. Adopting the same concept, we designed a new fingertip sensor with the structure that can be seen in Fig. 1. Here, we are using 24 MLX90393 chips mounted on a flexible PCB (Printed Circuit Board) (Fig. 2). Each MLX90393 chip can provide 3-axis magnetic data and 1 temperature data. A single chip has a 7-bit I2C address where the last 2 bit can be configured by connecting two of the chip's pins to either supply voltage (VDD) or ground (VSS). As a result, one I2C bus segment (SDA) can handle four chips at the same time. Our fingertip sensor has nine electrical lines in total (VDD, VSS, SCL (clock), and 6 SDAs) for transferring $96(24 \times(3+1))$ measurement data.

In the current implementation we use rather bulky electronics to collect the I2C measurements. However, small sized microcontrollers are available; for example, the microcontroller board used for the skin sensors in iCub is about 26x18x6 mm big, can collect measurements from four I2C buses, and is connected on a daisy chain CAN (Controller Area Network) bus [9] [26]. It can significantly reduce the number of wiring. We will also implement a similar system in the future so that covering a whole hand with minimal wiring can be achieved.

\section{B. Manufacturing Process}

The manufacturing process was divided into two steps. We produced the fingertip based on the same shape that was previously introduced in [1].

1) Electronics: Since the fingertip does not have a flat surface, we use a flexible PCB that can conform to the contour

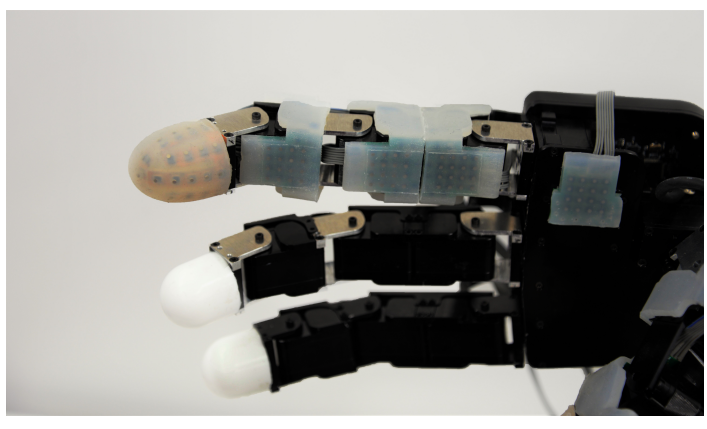

Fig. 4. Allegro hand covered with uSkin on its fingertip, phalanges, and palm.

of the surface. We designed a 3D model of the PCB in SolidWorks according to the shape of the fingertip. Later, the model was flattened producing a flat flexible PCB as in Fig. 2 (left). After all chips and cables had been soldered, the flexible PCB was glued to the 3D printed fingertip (Fig. 2 (right)).

2) Silicone skin: First, we manufactured the silicone mold using a $3 \mathrm{D}$ printer. Liquid silicone rubber was poured into the molding cast, and then the hole maker for placing magnets was inserted. After the silicone skin completely cured, we removed it from the molding cast and placed all 24 magnets inside the holes. The orientation was configured so that the south pole of the magnets faces the chips. The magnets for the current implementation were neodymium (grade N50) with a $1.59 \mathrm{~mm}$ diameter and a $0.53 \mathrm{~mm}$ thickness. It had an optimal pull of $226.8 \mathrm{~g}$ and 729 surface gauss. Next, the skin with embedded magnets was placed into another molding cast, and more liquid silicone rubber was poured to embed all of the magnets. Fig 3 . shows the complete manufacturing process of the uSkin fingertip silicone.

The material used was Ecoflex Supersoft with shore hardness 00-50 from Smooth-On. We also tried Ecoflex 30, but the material was too soft. A soft sensor can provide more sensitivity, however, the maximum range that can be measured will be reduced as a trade-off. In our case, with Ecoflex 30 only $5 \mathrm{~N}$ was needed to saturate the Hall-effect sensor. Therefore, Ecoflex 50 was purposely chosen to provide more range.

Finally, we covered the previously made 3D printed fingertip including the PCB with the silicone skin and then mounted it to the Allegro Hand as in Fig. 4. The figure also shows the skin for flat phalanges that has been presented in [7].

\section{EVALUATION}

Several experiments were conducted to estimate uSkin's performance. The first experiment is about calibrating and evaluating the sensor's characteristics. As uSkin has multiple sensing points distributed in 24 different locations, we presumed that this sensor can be used for identifying object shapes. We tested the distributed sensing and crosstalk response. Finally, we evaluated the sensor response while grasping objects.

\section{A. Experimental Setup}

The overall test setup used in this paper can be seen in Fig. 5. A current controlled voice coil motor without force 


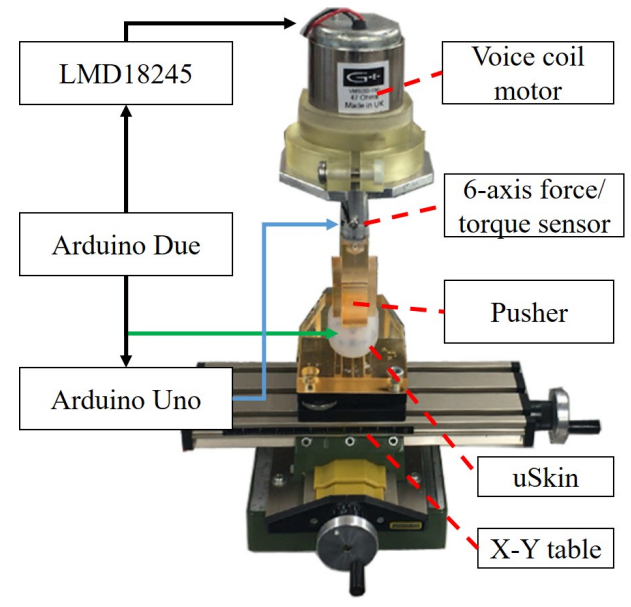

Fig. 5. Test setup used in this paper.

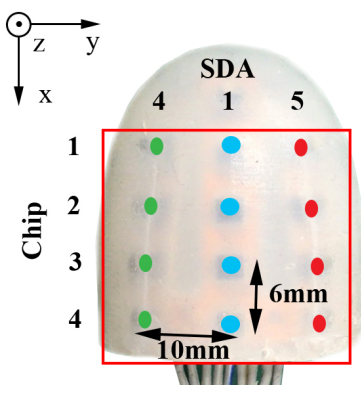

Fig. 6. SDA and chip placement.

feedback (VM5050-190 from Geeplus) was utilized to apply varying force to the skin sensor. The load force can be changed by controlling the current of the voice coil motor using a full-bridge motor driver (LMD18245 from Texas Instruments). A 6-axis force/torque (F/T) sensor (Nano 1.5/1.5 from BL autotech) is used for monitoring the amount of applied force. The maximum force that can be measured by this sensor is around $14 \mathrm{~N}$ for the $\mathrm{x}, \mathrm{y}$, and $\mathrm{z}$-axis. However, the maximum force value applied in this experiment was limited to only around $6 \mathrm{~N}$. The current fingertip structure has a higher sensitivity than the one previously made (uSkin for phalanges can measure up to $14 \mathrm{~N}$ [7]). Applying more than $7 \mathrm{~N}$ causes saturation. A 3D printed push plate was attached to the F/T sensor. We used different shapes depending on the task. For the normal and shear force calibration task, we used a flat pusher with a $12 \times 12 \mathrm{~mm}$ square contact area. For the shape recognition task, we used four different shapes $(5 \times 35 \mathrm{~mm}$ rectangle, $15 \times 15 \mathrm{~mm}$ square, $10 \mathrm{~mm}$ diameter circle, and $7 \times 7$ $\mathrm{mm}$ square).

The Nano 1.5/1.5 requires $5 \mathrm{~V}$ supply voltage to operate. Meanwhile, uSkin requires $3.3 \mathrm{~V}$. Because of these requirements, we used one Arduino Uno for recording the F/T sensor measurements and one Arduino Due for recording uSkin sensor measurements. The Arduino's were synchronized and both data were recorded on memory cards for offline analysis.

Each load cell (MLX90393) has a sampling rate that can be configured up to around $500 \mathrm{~Hz}$. As uSkin requires 6

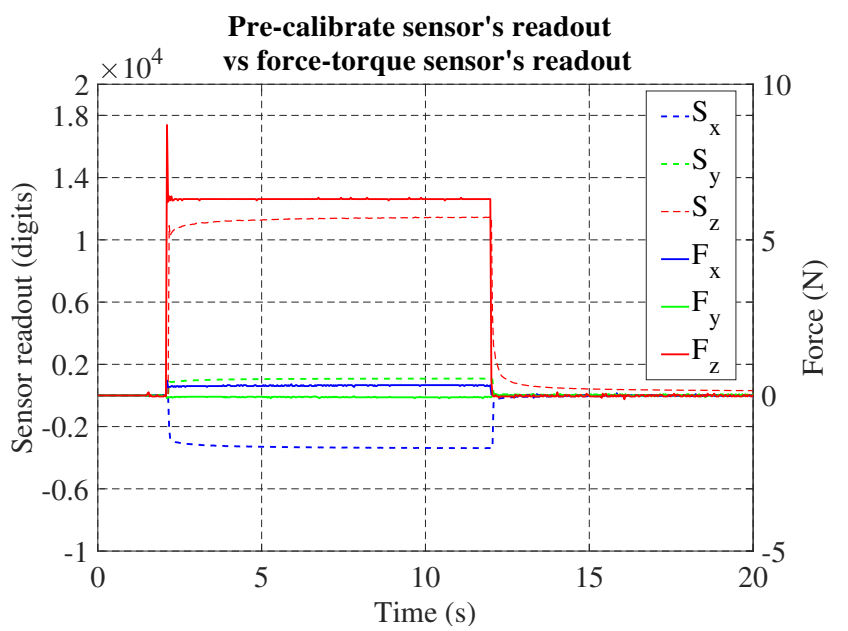

Fig. 7. uSkin's readout and the corresponding force from F/T sensor when only the normal force is applied ( $\mathrm{S}=$ sensor, $\mathrm{F}=$ reference sensor).

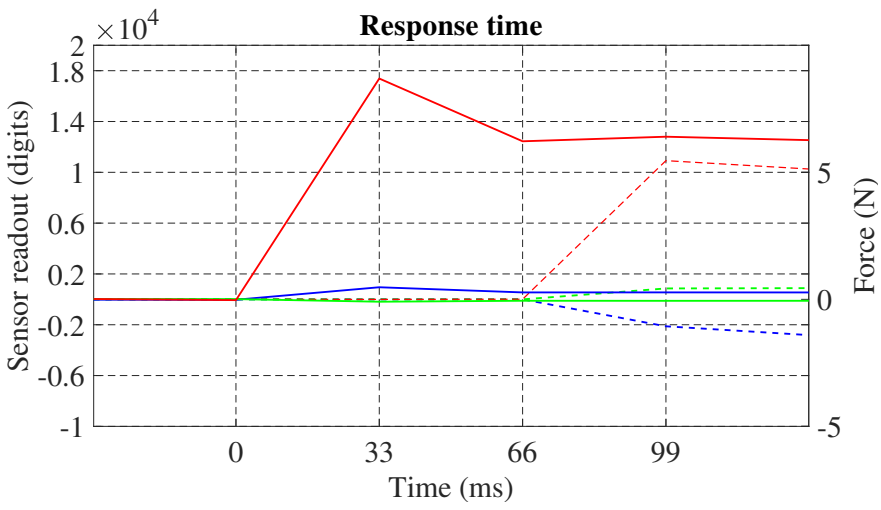

Fig. 8. Closer look of the response time.

data (SDA) lines to read all measurements, we used an I2C multiplexer (TCA9548A from Adafruit) connected to a single SDA pin of an Arduino Due. Therefore, the maximum sampling rate that we can achieve at the moment is limited to only around $30 \mathrm{~Hz}$. In the future, we plan to use smaller microcontrollers with multiple I2C ports.

The fingertip with the skin sensor was fixed on a sturdy X$\mathrm{Y}$ table; the position where to apply the force can be adjusted with the X-Y table; also shear force can be applied by moving the $\mathrm{X}-\mathrm{Y}$ table after an initial contact with the push plate. The sensor was mounted on a tiltable platform so that the voice coil motor applied perpendicular force to the skin surface.

The average of $1 \mathrm{~s}$ of each measurement when the sensor was not pushed was used as the baseline and subtracted from the measurements. Otherwise, unfiltered sensor data was used for all experiments and is shown in the plots.

\section{B. Sensor Measurements Before Calibration}

When only normal force was applied on the top of uSkin, there were also some magnet displacements in the $\mathrm{x}$-axis and $y$-axis (details of the orientation can be seen in Fig. 6). This can be seen by comparing the Hall effect sensor response (SDA 1 chip 2) to the reference sensor (Fig. 7). 


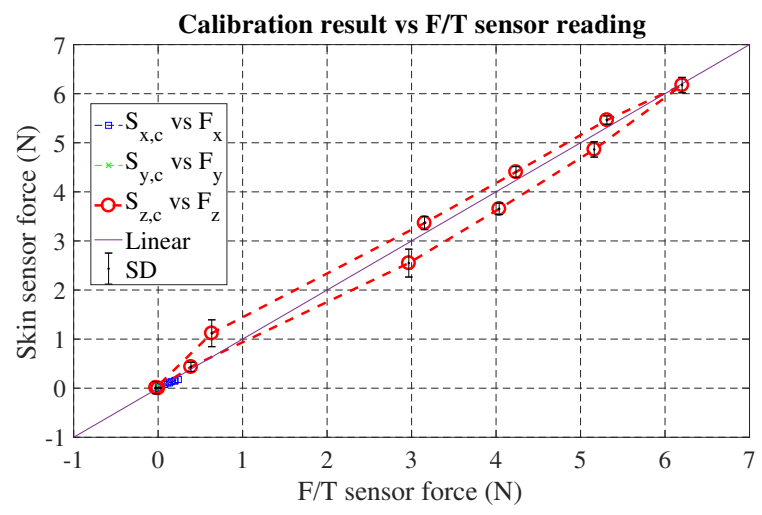

Fig. 9. Calibrated sensor response when normal force is applied.

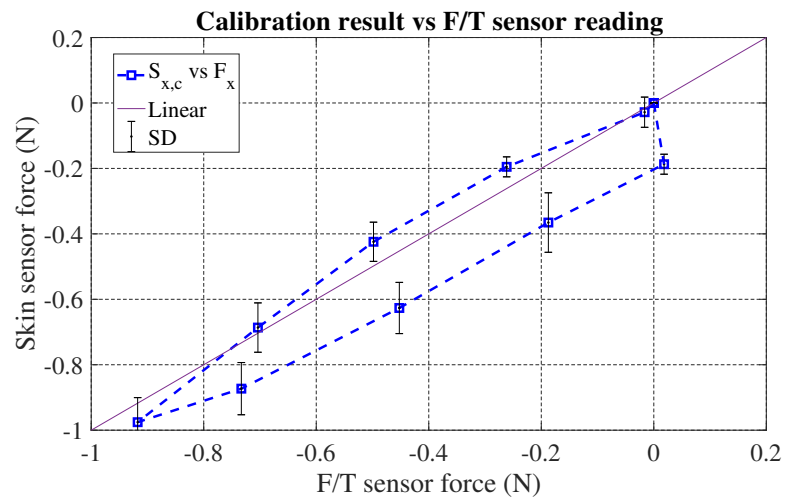

Fig. 10. Calibrated sensor response when shear force is applied (x-axis only).

It can be seen that there was no force in the $y$-axis in the $\mathrm{F} / \mathrm{T}$ sensor (Fy, marked as green). However, a magnet displacement was measured in this axis in uSkin (Sy, marked as green). This problem happened most likely because of the magnet placement which is not perfectly centered, or due to the incompressibility of the silicone material. For this reason, uSkin needs to be calibrated.

\section{Response time}

The sensor's response time can be seen in Fig. 8. The graph shows that uSkin can give a response in two time steps $(66 \mathrm{~ms})$ after it received an impact force. The sensor requires about one second to achieve its level state.

\section{Calibration}

1) Training set preparation: The sensor was calibrated with uSkin measurements as the input and reference sensor measurements as the target when applying force only in the $\mathrm{x}, \mathrm{y}$ or $\mathrm{z}$-axis, respectively. In particular, three kinds of force (normal, shear in the x-axis, and shear in y-axis) were applied on the top of each load cell, resulting in three time series data to calibrate each Hall effect sensor. For the normal force, each load cell was pushed for 4 seconds, then the pusher was retracted (no force applied) for 1 minute to ensure that the silicone skin had properly returned to its initial state before higher force was applied. Four different normal forces were

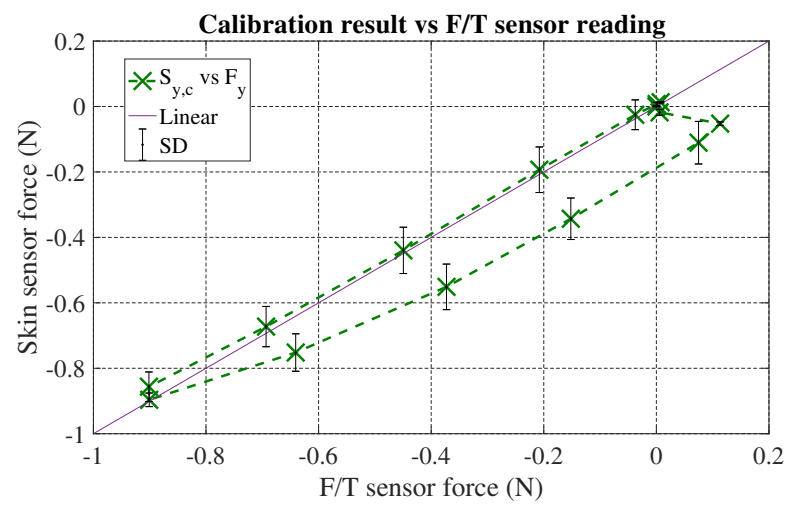

Fig. 11. Calibrated sensor response when shear force is applied (y-axis only).

applied to each load cell, of around $3 \mathrm{~N}$ (the voice coil motor has limitations for the lowest forces it can produce), $4 \mathrm{~N}, 5$ $\mathrm{N}$ and $6 \mathrm{~N}$. Five samples before and after each load change were removed to clean the training dataset from unwanted transient signals. By conducting in such a way, we found that the prediction performance for test data can be increased. For the shear forces, we applied a constant $3 \mathrm{~N}$ force in the $\mathrm{z}$-axis. To apply a shear force, we manually moved the X-Y table while the sensor was being pushed. We used a metronome to guide the timing. Every 4 seconds the fine adjuster knob was turned resulting in a $0.2 \mathrm{~mm}$ displacement either in $\mathrm{x}$ or $\mathrm{y}$ direction from the center of the load cell. After four steps had been performed the pusher was retracted.

2) Calibration parameters: In previous work, we found that calibrating our sensor with a quadratic model and Huber robust option can give a better prediction performance compared to linear regression or when using a neural network. For this reason, we also calibrated the fingertip load cells using the same method. For each chip, all three sensor measurements are used to calculate each force in the $\mathrm{x}, \mathrm{y}$, and $\mathrm{z}$-axis. Nine parameters for each axis have to be calculated using this formula:

$$
\begin{array}{r}
S_{j, c}=a S_{x}+b S_{y}+c S_{z}+d S_{x} * S_{y}+ \\
e S_{x} * S_{z}+f S_{y} * S_{z}+g S_{x}^{2}+h S_{y}^{2}+i S_{z}^{2}
\end{array}
$$

Here, $S_{j, c}$ is the calibrated sensor output of axis $j$ (x, y, or z). $S_{x}, S_{y}$, and $S_{z}$ are pre-calibrated skin sensor module outputs in digits. $a$ to $i$ are the calibration parameters. These parameters were calculated in MATLAB using the Statistics \& Machine Learning Toolbox.

3) Calibration and hysteresis result: The normal force test data was prepared as follows. In the first four seconds, the pusher was in a retracted position (no load force). In the next four seconds, the pusher was released, touching the surface of the uSkin. Afterwards, every four seconds, using a stepwise force, we increased the load four times and then decreased again four times. Finally, the force was unloaded (only touching the surface) for four seconds and then the pusher was retracted. In total, there were 11 steps. The input output graph of the calibrated skin sensor can be seen in Fig. 9. $S_{x, c}, S_{y, c}$, and $S_{z, c}$ are the calibrated skin sensor outputs in 
$\mathrm{x}, \mathrm{y}$, and $\mathrm{z}$-axis, respectively. Like in the previous sections, we used chip 2 on SDA1 (see Fig. 6). SD is the standard deviation and shown in black for easier visibility. It can be seen that the calibrated sensor measurements correspond to the reference sensor. We found that when there was no load force under a static condition (the sensor did not move), the sensor output varied in the range of $\pm 0.02 \mathrm{~N}$ or around $2 \mathrm{gf}$ (gram-force). Therefore, this is the threshold value of our current sensor. Furthermore, using equation 2, we found that the hysteresis of our sensor during the normal force load was $10 \%$.

$$
\text { Hysteresis } \%=\left|\frac{\left(F_{m u}-F_{m l}\right)}{\left(F_{\max }-F_{\min }\right)}\right| \times 100 \%
$$

$F_{m l}$ and $F_{m u}$ are the calibrated skin force values (linear interpolation of the nearest neighbors) of the loading and unloading cycles, respectively, taken at the midpoint force of $(6.3 \mathrm{~N}-0 \mathrm{~N}) / 2=3.15 \mathrm{~N} . F_{\min }$ was the minimum measured average force $(0 \mathrm{~N})$ and $F_{\max }$ the maximum measured average force $(6.3 \mathrm{~N})$ by the reference sensor.

For the shear forces test data, we used a similar method as for the training set preparation. However, after four steps displacing the chip from the center of the pusher, we also unload the shear force by stepwise returning the sensor to its initial position. The shear forces test results for $\mathrm{x}$-axis and $\mathrm{y}$ axis can be seen in Fig. 10 and Fig. 11, respectively. We can see from the graph that the skin sensor force in the end did not return to zero. This occurred most likely because of the hysteresis effect. To mitigate this, a special compensation is required. In the future, we will also use a motorized $\mathrm{X}-\mathrm{Y}$ table to make the timing and movements more precise.

\section{E. Signal to noise ratio}

In this experiment, we used the calibrated sensor output of the z-axis from the previous section to calculate its SNR (signal to noise ratio) value. The calculation can be done using equation 3 or 4 [27].

$$
\begin{gathered}
S N R=\frac{\left|\mu_{U}-\mu_{p}\right|}{\sigma_{u}} \\
S N R_{d B}=20 \log _{10}\left(\frac{\left|\mu_{U}-\mu_{p}\right|}{\sigma_{u}}\right) d B
\end{gathered}
$$

In these equations, $\mu_{U}$ is the mean value of the uSkin measurements when not loaded, $\mu_{p}$ is the average uSkin measurement when loaded, and $\sigma_{u}$ is the standard deviation value when not loaded. All SNR values were fitted using a MATLAB Curve Fitting Toolbox to create an SNR model. We used a two-term exponential model with a bisquare robust option. We found that this model can give a better fitting compared to the polynomial model. Fig. 12 shows the SNR model of the uSkin fingertip. Here, the first point represents the force produced by the weight of the pusher (no load force from the voice coil motor). The second point is the minimum load force that the voice coil motor can produce. Therefore, there is no data in between these two values. The SNR value was higher compared to our previous work [6]. In Table I, the SNR values of uSkin for the flat (i.e. our

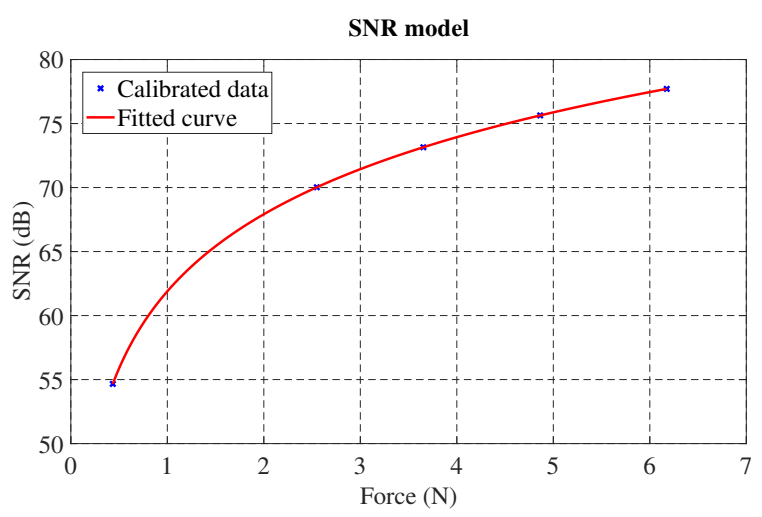

Fig. 12. SNR model of the uSkin for a fingertip.

previously published design [7]) and curved (i.e. fingertip, our new design introduced in this paper) phalange are provided. The SNR value of the phalange skin was calculated using its corresponding SNR model.

TABLE I

SNR COMPARISON VALUE.

\begin{tabular}{ccc}
\hline \hline \multirow{2}{*}{ Force $(\mathrm{N})$} & SNR $(\mathrm{dB})$ & \\
& Curved (fingertip) & Flat \\
\hline 0.43 & 54.66 & 29.41 \\
2.55 & 70.02 & 52.50 \\
3.65 & 73.14 & 54.39 \\
4.86 & 75.63 & 55.61 \\
6.17 & 77.70 & 56.57 \\
\hline \hline
\end{tabular}

\section{F. Visualization and crosstalk}

In the previous section, we showed that a load cell of uSkin can measure a three-dimensional force vector. Here, we visualize the response vectors when the fingertip was pressed by an object with a specific shape and various load forces. The pusher was placed centered above chip no. 3 SDA 1. As the pusher only covers an area in SDA 1, 4, and 5 (Fig. 6), we only plot the measurements from these chips. The sensor was pushed perpendicularly to the skin surface with stepwise force from around $0.5 \mathrm{~N}$ to $6.3 \mathrm{~N}$. The resulting ten measurement vectors for each chip are shown in Fig. 13. The colors of the vectors correspond to the SDAs shown in Fig. 6. We used different scaling to plot the vector arrows. For the $5 \times 35$ $\mathrm{mm}$ rectangular and $15 \times 5 \mathrm{~mm}$ square shapes, the output of chip no. 2-4 SDA 1 were scaled down with 1/8 ratio. For the round and $7 \times 7 \mathrm{~mm}$ rectangular shapes, only the output of chip no. 3 SDA 1 was scaled down with $1 / 8$ ratio. This is necessary to provide a clear visualization as the pressures produced by pushers with smaller contact area were higher. For the $5 \times 35 \mathrm{~mm}$ rectangular shape, we can see that chip no. 2-4 on SDA 1 produced the most significant response. The response in chip no. 1 is low most likely because of the curved fingertip shape. Due to the curvature of the fingertip, 

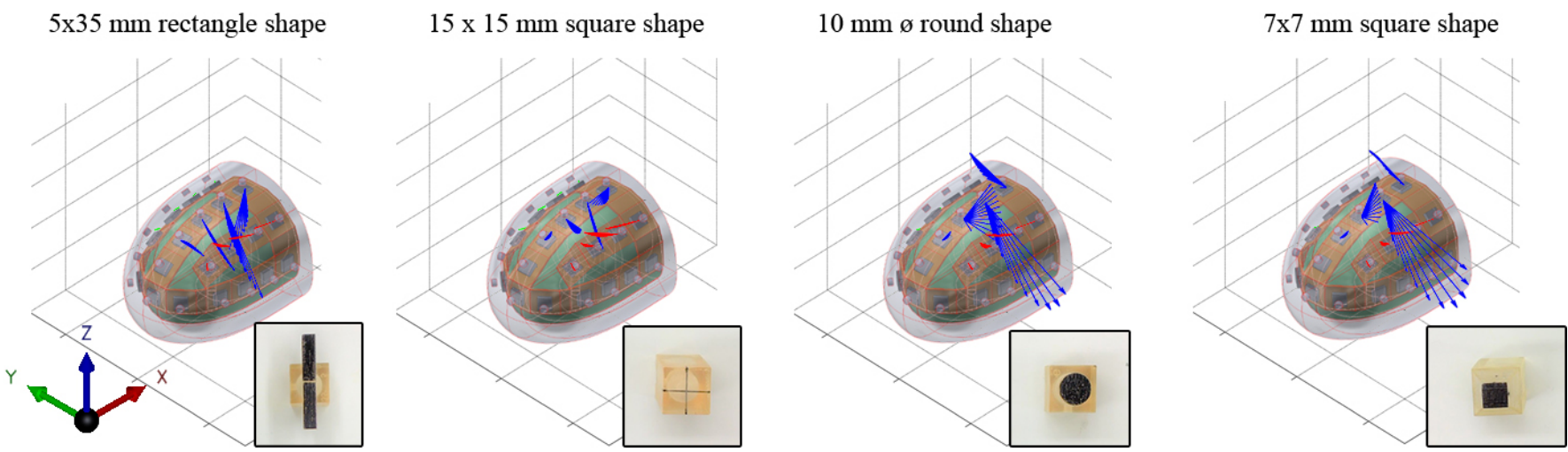

Fig. 13. Response vectors when different shapes and forces are applied on the sensor. The colors of the vectors correspond to the SDAs shown in Fig. 6 .

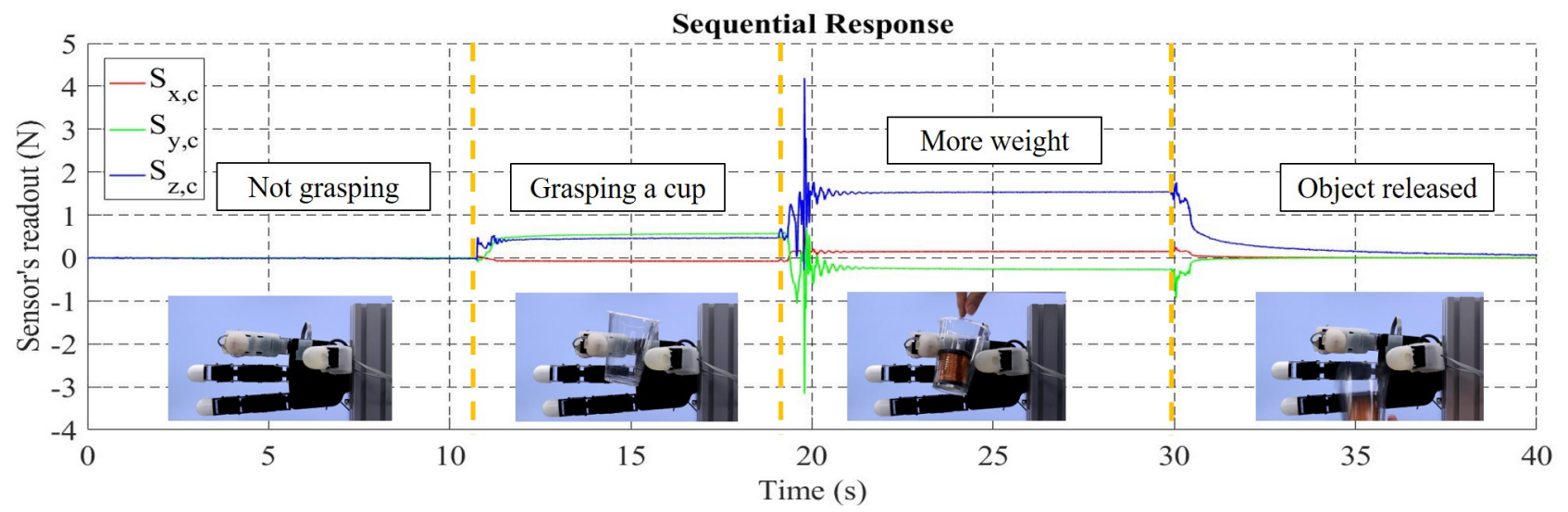

Fig. 14. Sequential response.

the pusher could not displace the magnet above chip no. 1 . We can also see that SDA 4 and 5 measured low force vectors (red and green arrows). Overall, the crosstalk effect produced from the magnetic field interference seems minimal and to not affect the performance of the sensor. The $15 \times 15 \mathrm{~mm}$ square shape has a lower response because it has a larger contact area compared to the rectangular shape. As for the round shape, we can see that only chip no. 3 has a positive response. The vector arrows of chip no. 2 and 4 became negative because of the silicone skin that was being pushed upwards. If we compare the response of the round and $7 \times 7 \mathrm{~mm}$ square shapes, there appears to be little difference. This makes sense as they have a similar shape and the size of the pusher is less than the distance between chip no. 2 and 4 . A higher spatial density would be required to easily detect the shape of a small sized object. In conclusion, uSkin can provide force vector data that might be used for tactile object recognition. However, the minimum shape size that can be detected is limited by the distance of each load cell (in this case $6 \mathrm{~mm}$ between each chip in the same SDA line and $10 \mathrm{~mm}$ between SDA lines).

\section{G. Implementation on the Allegro Hand}

In this section, we will show uSkin's response when it is implemented on the Allegro Hand. The output of one load cell (SDA 1 chip 2) corresponding to different grasping conditions can be seen in Fig. 14. Initially, the Allegro Hand did not grasp anything so there was no response from the sensor. When the hand grabbed the cup, measurements in $\mathrm{x}, \mathrm{y}$, and $\mathrm{z}$-axis changed. Later, we dropped a $175 \mathrm{~g}$ heavy roll of wires into the cup, which caused a visible vibration and the cup slightly moved due the weight of the object. The output of uSkin changed accordingly as shown in the plot. Finally, the cup was released and the output of the sensor eventually returned to zero.

\section{CURRENT LIMITATIONS}

As our sensor uses magnetic field changes induced by the skin deformation as its sensing principle, different object materials, in particular ferromagnetic objects, can potentially affect the measurement. Also our preliminary work showed that different kinds of material that are in contact with the sensor may affect its output response [6]. For compensating the influence of the external magnetic field or material properties, a reference sensor could be used. Similarly, the earth magnetic field influences the sensor, but again this could be compensated with a reference sensor or by calculating the sensor orientation using the robot kinematics. Moreover, we see the purpose of our sensor in that it enables to study the importance of distributed force vector sensing in a soft skin for tasks like object manipulation and object shape recognition of grasped objects. We think that certain limitations (like using objects made only from plastics) are reasonable for this purpose. 


\section{CONCLUSIONS}

This paper presented the design of a skin sensor for robot fingertips with 24 Hall effect sensors. Load tests were performed by applying normal and shear forces on the proposed sensor. The tests revealed that even though it has a round shape, uSkin can successfully be calibrated and measure 3-axis force. Further tests were performed to measure the distributed sensor response when being pushed with different shapes. It was concluded that the sensor can be used to detect the distributed force vector. In future work, we want to include an air gap between the chip and the magnet to counteract the incompressibility of the silicone and its associated effects.

\section{ACKNOWLEDGMENT}

This research was partially supported by the JSPS Grant-inAid for Scientific Research (S) No. 25220005, JSPS Grant-inAid for Young Scientists (B) No. 15K21443 \& 17K18183, Research Institute for Science and Engineering of Waseda University, the Program for Leading Graduate Schools, Graduate Program for Embodiment Informatics of the Ministry of Education, Culture, Sports, Science and Technology, and Program for Creating STart-ups from Advanced Research and Technology ST282005NO.

\section{REFERENCES}

[1] K. Or, M. Tomura, A. Schmitz, S. Funabashi, and S. Sugano, "Positionforce combination control with passive flexibility for versatile inhand manipulation based on posture interpolation," in 2016 IEEE/RSJ International Conference on Intelligent Robots and Systems (IROS), Oct 2016, pp. 2542-2547.

[2] H. Iwata and S. Sugano, "Design of human symbiotic robot TWENDYONE," in Robotics and Automation, 2009. ICRA'09. IEEE International Conference on. IEEE, 2009, pp. 580-586.

[3] Z. Kappassov, J.-A. Corrales, and V. Perdereau, "Tactile sensing in dexterous robot hands-Review," Robotics and Autonomous Systems, vol. 74, pp. 195-220, 2015.

[4] S. Somlor, R. S. Hartanto, A. Schmitz, and S. Sugano, "A novel triaxial capacitive-type skin sensor," Advanced Robotics, vol. 29, no. 21, pp. 1375-1391, 2015.

[5] T. P. Tomo, S. Somlor, A. Schmitz, L. Jamone, W. Huang, H. Kristanto, and S. Sugano, "Design and characterization of a three-axis hall effectbased soft skin sensor," Sensors, vol. 16, no. 4, p. 491, 2016.

[6] T. P. Tomo, W. K. Wong, A. Schmitz, H. Kristanto, S. Somlor, J. Hwang, and S. Sugano, "SNR modeling and material dependency test of a lowcost and simple to fabricate 3D force sensor for soft robotics," in System Integration (SII), 2016 IEEE/SICE International Symposium on. IEEE, 2016, pp. 428-433.

[7] T. P. Tomo, W. K. Wong, A. Schmitz, H. Kristanto, A. Sarazin, L. Jamone, S. Somlor, and S. Sugano, "A modular, distributed, soft, 3axis sensor system for robot hands," in Humanoid Robots (Humanoids), 2016 IEEE-RAS 16th International Conference on. IEEE, 2016, pp. 454-460.

[8] K. Or, S. Morikuni, S. Ogasa, S. Funabashi, A. Schmitz, and S. Sugano, "A study on fingertip designs and their influences on performing stable prehension for robot hands," in Humanoid Robots (Humanoids), 2016 IEEE-RAS 16th International Conference on. IEEE, 2016, pp. 772-777.
[9] A. Schmitz, P. Maiolino, M. Maggiali, L. Natale, G. Cannata, and G. Metta, "Methods and technologies for the implementation of largescale robot tactile sensors," IEEE Transactions on Robotics, vol. 27, no. 3, pp. 389-400, 2011 .

[10] R. Koiva, M. Zenker, C. Schürmann, R. Haschke, and H. J. Ritter, "A highly sensitive 3d-shaped tactile sensor," in Advanced Intelligent Mechatronics (AIM), 2013 IEEE/ASME International Conference on. IEEE, 2013, pp. 1084-1089.

[11] R. S. Dahiya, G. Metta, M. Valle, and G. Sandini, "Tactile sensing-from humans to humanoids," IEEE Transactions on Robotics, vol. 26, no. 1, pp. 1-20, 2010.

[12] R. S. Dahiya, P. Mittendorfer, M. Valle, G. Cheng, and V. J. Lumelsky, "Directions toward effective utilization of tactile skin: A review," IEEE Sensors Journal, vol. 13, no. 11, pp. 4121-4138, 2013.

[13] T. Mouri, H. Kawasaki, K. Yoshikawa, J. Takai, and S. Ito, "Anthropomorphic robot hand: Gifu hand III," in Proc. Int. Conf. ICCAS, 2002, pp. 1288-1293.

[14] T. Yoshikai, M. Hayashi, Y. Ishizaka, H. Fukushima, A. Kadowaki, T. Sagisaka, K. Kobayashi, I. Kumagai, and M. Inaba, "Development of robots with soft sensor flesh for achieving close interaction behavior," Advances in Artificial Intelligence, vol. 2012, p. 8, 2012.

[15] W. Yuan, R. Li, M. A. Srinivasan, and E. H. Adelson, "Measurement of shear and slip with a GelSight tactile sensor," in Robotics and Automation (ICRA), 2015 IEEE International Conference on. IEEE, 2015, pp. 304-311.

[16] B. Winstone, G. Griffiths, T. Pipe, C. Melhuish, and J. Rossiter, "TACTIP-tactile fingertip device, texture analysis through optical tracking of skin features," in Conference on Biomimetic and Biohybrid Systems. Springer, 2013, pp. 323-334.

[17] T. Zhang, L. Jiang, X. Wu, W. Feng, D. Zhou, and H. Liu, "Fingertip three-axis tactile sensor for multifingered grasping," IEEE/ASME Transactions on Mechatronics, vol. 20, no. 4, pp. 1875-1885, 2015.

[18] J. J. Clark, "A magnetic field based compliance matching sensor for high resolution, high compliance tactile sensing," in Robotics and Automation, 1988. Proceedings., 1988 IEEE International Conference on. IEEE, 1988, pp. 772-777.

[19] W. C. Nowlin, "Experimental results on Bayesian algorithms for interpreting compliant tactile sensing data," in Robotics and Automation, 1991. Proceedings., 1991 IEEE International Conference on. IEEE, 1991, pp. 378-383.

[20] L. Jamone, G. Metta, F. Nori, and G. Sandini, "James: A humanoid robot acting over an unstructured world," in Humanoid Robots, 2006 6th IEEE-RAS International Conference on. IEEE, 2006, pp. 143-150.

[21] L. Jamone, L. Natale, G. Metta, and G. Sandini, "Highly sensitive soft tactile sensors for an anthropomorphic robotic hand," IEEE sensors Journal, vol. 15 , no. 8, pp. 4226-4233, 2015.

[22] C. Ledermann, S. Wirges, D. Oertel, M. Mende, and H. Woern, "Tactile sensor on a magnetic basis using novel 3D Hall sensor-First prototypes and results," in Intelligent Engineering Systems (INES), 2013 IEEE 17th International Conference on. IEEE, 2013, pp. 55-60.

[23] E. Torres-Jara, I. Vasilescu, and R. Coral, "A soft touch: Compliant tactile sensors for sensitive manipulation," http://hdl.handle.net/1721.1/31220/, 2006, [Online; accessed 18May-2017].

[24] S. Youssefian, N. Rahbar, and E. Torres-Jara, "Contact behavior of soft spherical tactile sensors," IEEE sensors Journal, vol. 14, no. 5, pp. 14351442, 2014.

[25] T. Paulino, P. Ribeiro, M. Neto, S. Cardoso, A. Schmitz, J. Santos-Victor, A. Bernardino, and L. Jamone, "Low-cost 3-axis soft tactile sensors for the human-friendly robot vizzy," in IEEE International Conference on Robotics and Automation, 2017.

[26] G. Cannata, M. Maggiali, G. Metta, and G. Sandini, "An embedded artificial skin for humanoid robots," in Multisensor Fusion and Integration for Intelligent Systems, 2008. MFI 2008. IEEE International Conference on. IEEE, 2008, pp. 434-438.

[27] B. Davison, "Techniques for robust touch sensing design," AN1334 Microchip Technology Inc, p. 53, 2010. 